\title{
AUTOESTIMA Y COMPRENSIÓN DE INFORMACIÓN EN EL ÁREA DE CIENCIA, TECNOLOGÍA Y AMBIENTE DEL PRIMER GRADO DE SECUNDARIA DE LAS INSTITUCIONES EDUCATIVAS ESTATALES DEL DISTRITO DE HUANCAYO*
}

\author{
Gómez Flores, Juan'; Rafaele De la Cruz, Mauro² y Vilca Loyza, Hugo³
}

\section{RESUMEN}

La investigación ha tenido como objetivo analizar la relación entre autoestima y el desarrollo de la capacidad de comprensión de información de los alumnos del primer grado de educación secundaria de las instituciones educativas de gestión estatal del distrito de Huancayo y la hipótesis: existe relación directa y significativa entre autoestima y el logro de la capacidad de comprensión de información en el Área de Ciencia, Tecnología y Ambiente por los alumnos del primer grado de secundaria de las instituciones educativas estatales del distrito de Huancayo. El método empleado fue el descriptivo, con diseño descriptivo correlacional; para la recolección de la información se utilizaron las técnicas de fichaje, psicometría y evaluación educativa con los instrumentos: fichas bibliográficas, de resumen y de trascripción textual, inventario de autoestima de Coopersmith y prueba pedagógica. La población estuvo constituida por 3669 alumnos y la muestra por 310 alumnos elegidos por muestreo aleatorio simple. Los resultados indican que los alumnos presentan un nivel alto de autoestima $(64,2 \%)$ y un nivel bajo de comprensión de información $(57,7 \%)$ y, que existe una correlación significativa $(p<0,05)$ directa pero baja entre autoestima y la capacidad de comprensión de información en el Área de Ciencia, Tecnología y Ambiente en los alumnos del primer grado de educación secundaria de las instituciones educativas de gestión estatal del distrito de Huancayo, de acuerdo con la prueba Z de Gauss, al $95 \%$ de confiabilidad.

Palabras clave: autoestima, comprensión de información, ciencia, tecnología y ambiente.

\section{SELF-ESTEEM AND UNDERSTANDING OF INFORMATION IN THE SCIENCE AREA, TECHNOLOGY AND ATMOSPHERE OF THE FIRST GRADE OF SECONDARY OF THE STATE EDUCATIONAL INSTITUTIONS OF THE DISTRICT OF HUANCAYO}

\begin{abstract}
The investigation has had as objective to analyze the relationship between self-esteem and the development of the capacity of understanding of the students' of the educational institutions of state administration of the district of Huancayo Information and the hypothesis; direct and significant relationship exists between self-esteem and the achievement of the capacity of understanding of information in the Area of Science, Technology and Atmosphere for the students of the first degree of secondary of the state educational institutions of the district of Huancayo. The used method was the descriptive one, with design descriptive correlacional; for the gathering of the information the fichaje techniques, psicometría and educational evaluation and the instruments were used: you register bibliographical, of summary and textual trascripción, test of Coopersmith and pedagogic test; the population was constituted by 3669 students and the sample by 310 elected students for simple aleatory sampling. The results indicate that a significant correlation exists $(p<0,05)$ direct and low between self-esteem and the capacity of understanding of the student's information; as well as according to gender and age according to the test $Z$ of Gauss to $95 \%$ of dependability.
\end{abstract}

Key words: self-esteem, understanding of information, science, technology and atmosphere.

* Este trabajo de investigación fue recibido el 20/03/2008 retornando para su revisión el 22/09/2008 y aprobado para su publicación el 27/02/2009.

1. Email: jbgomez£@yahoo.es

2. Email:maurorafaele@hotmail.com

3. Email: hugovilca15@hotmail.com 


\section{INTRODUCCIÓN}

La investigación de la autoestima y comprensión de información en el Área de Ciencia, Tecnología y Ambiente de los alumnos del primer grado de secundaria de las instituciones educativas estatales del distrito de Huancayo; ha buscado respuesta al problema ¿cuál es la relación entre autoestima y el logro de la capacidad de comprensión de información en el Área de Ciencia, Tecnología y Ambiente en los alumnos del primer grado de secundaria de las instituciones educativas estatales del distrito de Huancayo? La autoestima es un juicio de valor sobre uno mismo y es educable no sólo en los primeros años, sino a lo largo de la vida. Algunos autores proponen que la autoestima determina el nivel de aprendizaje $y$, por ende, los alumnos con alta autoestima tienen mejor nivel de comprensión de información, etc.

Destacan las investigaciones de Bardales (1993), determinó que no existe correlación significativa entre la autoestima y el rendimiento académico, en función al tipo de familia a la que pertenece el estudiante de primaria; los alumnos que viven con sus padres tienen mayor autoestima que los que viven sólo con uno de ellos y, a su vez, éstos mayor que los que viven sin padres; Taramona (1987) demostró que existe relación significativa en la medida en que a mayor autoestima existe menor dogmatismo y mayor rendimiento académico; Branden (1993) afirmó que la autoestima es la experiencia de ser aptos para la vida y para las necesidades de la vida; Gómez y Vilca (2004) determinaron que la autoestima influye significativamente en el rendimiento escolar de los niños de once años de edad de las escuelas estatales urbano-marginal y rural del distrito de El Tambo; Gómez, Rafaele y Vilca (2005) comprobaron que la autoestima influye significativamente en el rendimiento académico de los estudiantes de la UNCP; estos últimos investigadores en el año 2006 determinaron la existencia de una relación media, directa y altamente significativa entre la autoestima y el rendimiento académico de los estudiantes por áreas profesionales de la UNCP.

La investigación realizada es importante porque toda persona sin una sana autoestima no puede realizarse en todo su potencial. La autoestima le permite adquirir la capacidad de asumir y vencer riesgos, adquirir nuevas aptitudes, ser líderes, y conducen al alumno y cualquier persona a los éxitos en la formación y desarrollo de sus habilidades potenciales e innatas. El impacto social de la investigación es positivo para los docentes, las autoridades y los alumnos porque es bueno que conozcan que el desarrollo de las capacidades intelectuales, motoras, etc., guarda una relación directa con el nivel de autoestima.

La investigación ha propuesto la hipótesis de que existe una relación directa y significativa entre autoestima y el desarrollo de la capacidad de comprensión de información en el Área de Ciencia, Tecnología y Ambiente en los alumnos del primer grado de secundaria de las instituciones educativas estatales del distrito de Huancayo; el objetivo fue analizar la relación entre autoestima y logro de la capacidad de comprensión de información en el Área de Ciencia, Tecnología y Ambiente. Se concluye que los alumnos presentan un nivel alto de autoestima $(64,2 \%)$ y un nivel bajo de comprensión de información $(57,7 \%)$ $y$, que existe una correlación significativa $(p<0,05)$ directa pero baja entre autoestima y la capacidad de comprensión de información de los alumnos de las instituciones educativas estatales del distrito de Huancayo.

\section{MATERIAL Y MÉTODOS}

La población estuvo constituida por 3669 alumnos matriculados en el primer grado de educación secundaria, matriculados en el año escolar 2007 y la muestra aleatoria por 310 alumnos elegidos de las instituciones educativas estatales Santa Isabel, Nuestra Señora de Cocharcas, María Inmaculada, José Carlos Mariátegui, Santa María Reyna y José Abelardo Quiñones. Se han empleado las técnicas del fichaje, la psicometría y la evaluación educativa, con sus instrumentos las diversas clases de fichas, el inventario de autoestima de Coopersmith y la prueba pedagógica, respectivamente.

Los datos de la autoestima se recopilaron aplicando el inventario de autoestima de Coopersmith y los referidos a la capacidad de comprensión de información mediante la prueba pedagógica, y fueron procesados con los softwares estadísticos MINITAB v_14, SPSS v_15 y Excel v_2003. 
Se ha empleado el método descriptivo con el diseño descriptivo-correlacional cuyo esquema es:

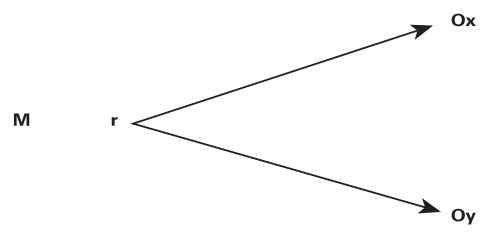

Donde:

M : es la muestra de estudio.

Ox : es la observación del nivel de autoestima.

Oy : esla información delacapacidad de comprensión de información en CTA.

XeY: representan la autoestima y la capacidad de comprensión de información en CTA.

$r$ : es la relación o correlación entre la autoestima y la capacidad de comprensión de información en CTA.

\section{RESULTADOS}

\section{Caracterización de la autoestima}

Tabla 1. Estadísticos de las dimensiones de la autoestima (n $=310$ ).

\begin{tabular}{lcc}
\hline \multicolumn{1}{c}{ Dimensión } & Media & DT \\
\hline Si mismo general & 0,68 & 0,169 \\
(SMG) & 0,61 & 0,215 \\
Social (SOC) & 0,73 & 0,237 \\
Hogar (HOG) & 0,68 & 0,233 \\
Colegio (COL) & & \\
Autoestima & 0,68 & 0,158 \\
\hline
\end{tabular}

En la tabla 1 se observa que la autoestima de los alumnos del primer grado de educación secundaria alcanza una media $(0,68)$ con una desviación típica de 0,158 . Por otro lado, las dimensiones de la autoestima también logran medias superiores al promedio ideal, donde la dimensión social logra la media menor $(0,61)$ y la dimensión hogar la media mayor $(0,73)$.

Tabla 2. Niveles de la autoestima.

\begin{tabular}{lcc}
\hline Nivel & Alumnos & $\%$ \\
\hline Baja & 11 & 3,5 \\
Media & 100 & 32,3 \\
Alta & 199 & 64,2 \\
Total & 310 & 100,0 \\
\hline
\end{tabular}

$Z c=11,68 ; p=0$
En la tabla 2 se aprecia que el $64,2 \%$ de los alumnos presentan una alta autoestima $(0,66$ a 1), el $32,3 \%$ una autoestima media $(0,35$ a 0,65$)$ y el $3,5 \%$ una baja autoestima do $(0$ a 0,34$)$.

Estos resultados comprueban la primera hipótesis específica, que al $99 \%$ de confianza estadística, los alumnos presentan una alta autoestima $(p=0,01)$, tal como se demuestra con la prueba $Z$ de Gauss para una proporción.

\section{Caracterización de la comprensión de información}

Tabla 3. Estadísticos de las capacidades específicas de la comprensión de información ( $n=310$ ).

\begin{tabular}{lcc}
\hline $\begin{array}{l}\text { Capacidad } \\
\text { específica }\end{array}$ & Media & DT \\
\hline Identifica (ID) & 2,66 & 1,142 \\
Interpreta (IN) & 1,76 & 1,476 \\
Describe (DE) & 2,00 & 2,003 \\
Discrimina (DI) & 2,00 & 2,003 \\
Analiza (AN) & 1,16 & 2,115 \\
Comprensión & 9,58 & 6,458 \\
\hline
\end{tabular}

En la tabla 3 se observa que la comprensión de información de los alumnos en el Área de Ciencia Tecnología y Ambiente (CTA) logra una media de 9,58 (48 \% de 20) con una desviación típica de 6,458. En la capacidad específica "identifica" la media es de 2,66 (67\% de 4) con una desviación estándar de 1,142; en la capacidad "interpreta" la media es de 1,76 (59\% de 3) con una desviación típica de 1,476; en la capacidad de "describe" la media es de 2 (50\% de 4) con una desviación estándar de 2,003; en la capacidad "discrimina" la media es de 2 (50\% de 4) con una desviación estándar de 2,003; en la capacidad "analiza" la media es de 1,16 (23\% de 5) con una desviación estándar de 2,115.

Tabla 4. Nivel de la comprensión de información.

\begin{tabular}{lcc}
\hline Nivel & Alumnos & $\%$ \\
\hline Baja & 179 & 57,7 \\
Media & 74 & 23,9 \\
Alta & 57 & 18,4 \\
Total & 310 & 100,0 \\
\hline
\end{tabular}

$Z c=9,26 ; p=0$ 
En la tabla 4 se observa que el $57,7 \%$ de los alumnos presentan una baja comprensión de información 10 a $10)$, el $23,9 \%$ una comprensión media (11 a 15) y el $18,4 \%$ una comprensión alta (16 a 20). Estos resultados comprueban la segunda hipótesis específica, que al $99 \%$ de confianza estadística, los alumnos del primer grado de secundaria de las instituciones educativas estatales de Huancayo presentan una baja comprensión de información en el área de Ciencia, Tecnología y Ambiente ( $p$ 0,01), tal como se comprueba con la prueba $Z$ de Gauss para una proporción.

\section{Relación entre autoestima y comprensión de información.}

Tabla 5. Coeficientes de correlación $r$ de Pearson entre autoestima y comprensión de información, por dimensiones de autoestima $(n=310)$.

\begin{tabular}{|c|c|c|c|c|c|c|}
\hline \multirow{2}{*}{$\begin{array}{l}\text { Comprensiór } \\
\text { de } \\
\text { información }\end{array}$} & \multirow{2}{*}{ Medidas } & \multicolumn{4}{|c|}{ Autoestima } & \multirow[b]{2}{*}{ Total } \\
\hline & & SMG & SOC & HOG & $\mathrm{COL}$ & \\
\hline \multirow[t]{2}{*}{ Identifica } & r & $0,172^{* *}$ & $0,158^{\star *}$ & 0,030 & 0,056 & $0,151^{\star \star}$ \\
\hline & p valor & 0,002 & 0,005 & 0,597 & 0,325 & 0,008 \\
\hline \multirow[t]{2}{*}{ Interpreta } & $r$ & $0,202^{* *}$ & 0,099 & 0,037 & 0,012 & $0,146^{*}$ \\
\hline & p valor & 0,000 & 0,081 & 0,520 & 0,8 & 0,010 \\
\hline \multirow[t]{2}{*}{ Describe } & $\mathrm{r}$ & $0,129^{*}$ & 0,064 & 0,053 & $-0,095$ & 0,076 \\
\hline & p valor & 0,023 & 0,261 & 0,355 & 0,094 & 0,181 \\
\hline \multirow[t]{2}{*}{ Discrimina } & r & 0,073 & 0,068 & 0,060 & $-0,033$ & 0,062 \\
\hline & p valor & 0,198 & 0,234 & 0,296 & 0,564 & 0,274 \\
\hline \multirow[t]{2}{*}{ Analiza } & $r$ & 0,070 & 0,111 & 0,035 & $-0,095$ & 0,049 \\
\hline & p valor & 0,217 & 0,050 & 0,538 & 0,095 & 0,386 \\
\hline \multirow{2}{*}{ Total } & $r$ & 0,162 & $0,128^{*}$ & 0,060 & $-0,058$ & $0,119^{*}$ \\
\hline & & & 0,024 & 0,292 & 0,308 & 0,036 \\
\hline
\end{tabular}

En la tabla 5 se observa que, al $95 \%$ de confianza estadística, existe correlación significativa $(p=0,05)$ directa y baja entre autoestima y comprensión de información de los alumnos $(r=0,119)$, tal como lo revela la prueba $Z$ de Gauss para el coeficiente de correlación lineal de Pearson.

De igual manera, existe correlación significativa (p 0,05) directa y baja entre la capacidad específica "interpreta" de comprensión de información y autoestima $(r=0,146)$ $y$, correlación altamente significativa $(p=0,01)$ entre la capacidad "identifica" y autoestima $(r=0,151)$.
Asimismo, existe correlación significativa (p 0,05 ) directa y baja entre la dimensión Social de autoestima y comprensión de información $(r=0,128)$ y, correlación altamente significativa $(p=0,01)$ entre la dimensión Sí mismo general de autoestima y comprensión de información $(r=0,162)$. Por otro lado, se aprecia correlación significativa $(p \quad 0,05)$ directa y baja entre la dimensión si mismo de autoestima y la capacidad "describe" ( $r=0,129)$. Además, se constatan correlaciones altamente significativas $(p$ $=0,01$ ) entre algunas dimensiones de autoestima y algunas capacidades específicas de comprensión de información, tales como entre la dimensión Sí mismo general y las capacidades "identifica" $(r=0,172$, directa y baja), "interpreta" ( $r=0,202$, directa y moderada), entre la dimensión social y la capacidad "identifica" ( $r$ $=0,158$, directa y baja).

Con estos resultados se comprueba la hipótesis general de investigación que plantea que "Existe relación entre autoestima y comprensión de información en el área de Ciencia, Tecnología y Ambiente del primer grado de educación secundaria de las instituciones educativas estatales del distrito de Huancayo".

\section{DISCUSIÓN}

La investigación realizada ha tenido como finalidad estudiar la relación entre la autoestima y la comprensión de información en el Área de Ciencia, Tecnología y Ambiente en los alumnos del primer grado de secundaria de las instituciones educativas estatales del distrito de Huancayo, en una muestra representativa de 310 alumnos de las instituciones educativas estatales del nivel secundario del distrito de Huancayo, comprensión UGEL-Huancayo, de la provincia de Huancayo, región Junín. Los resultados de la aplicación del Inventario de Autoestima de Coopersmith permiten afirmar que el $64,2 \%$ de alumnos presentaron una alta autoestima (0,66 a 1), el 32,3\% autoestima media $(0,35$ a 0,65$)$ y el $3,5 \%$ una baja autoestima $(0$ a 0,34$)$.

En la capacidad de comprensión de información del Área de Ciencia Tecnología y Ambiente (CTA), los alumnos lograron un promedio de 9,58 (48 \% de 20); en la capacidad específica "identifica", 2,66 (67\% de 4); en la capacidad "interpreta", 1,76 (59\% de 3); en la capacidad "describe", 2 (50\% de 4); en la 
capacidad "discrimina", 2 (50\% de 4) y en la capacidad "analiza", de 1,16 (23\% de 5). Asimismo, los resultados muestran que el $57,7 \%$ de los alumnos presentan una baja comprensión de información (0 a 10), el 23,9\% una comprensión media (11 a 15) y el 18,4 \% una comprensión alta (16 a 20).

Bardales (1993) determinó que no existe correlación significativa entre la autoestima y el rendimiento académico en función al tipo de familia a la que pertenece el estudiante de primaria; Taramona (1987) demostró que existe una relación significativa en la medida en que a mayor autoestima hay menor dogmatismo y mayor rendimiento académico; Gómez y Vilca (2004) determinaron que la autoestima influye significativamente en el rendimiento escolar de los niños de once años de edad de las escuelas estatales urbano-marginales y rurales del distrito de El Tambo; Gómez, Rafaele y Vilca (2005) comprobaron que la autoestima influye significativamente en el rendimiento académico de los estudiantes de la UNCP; los mismos investigadores (2006) concluyeron que existe una relación media, directa y altamente significativa entre la autoestima y el rendimiento académico de los estudiantes según áreas profesionales de la UNCP.

El estudio realizado corrobora con los trabajos mencionados líneas arriba, porque llega a la conclusión de que existe correlación significativa, directa y baja entre autoestima y comprensión de información de los alumnos del primer grado de secundaria, tal como lo confirma la prueba $Z$ de Gauss para el coeficiente de correlación lineal de Pearson.

De igual manera, existe correlación significativa, directa y baja entre las capacidades específicas "interpreta" e "identifica" con autoestima; entre la dimensión "social" y "sí mismo general" con comprensión de información; entre la dimensión "sí mismo general" con las capacidades específicas "describe", "identifica" e "interpreta"; y, entre la dimensión "social" y la capacidad "identifica".

Con los resultados obtenidos se ha comprobado la hipótesis de investigación planteada: "existe relación directa y significativa entre autoestima y la capacidad de comprensión de información en el Área de Ciencia, Tecnología y Ambiente en los alumnos del primer grado de educación secundaria de las instituciones educativas estatales del distrito metropolitano de Huancayo".

Los resultados indican con claridad que ambas variables de estudio se relacionan directa $y$ significativamente entre autoestima y comprensión de información, hallazgo que permite sostener que no se debe descuidar la autoestima de los alumnos para un logro adecuado de las capacidades; lo contrario devendría en situaciones poco alentadoras para el trabajo educativo, sabiendo que la autoestima es la clave para el éxito y la superación personal.

\section{CONCLUSIONES}

- El 64,2 \% de los alumnos del primer grado de educación secundaria de las instituciones educativas estatales del distrito de Huancayo presenta un nivel alto de autoestima $(0,66$ a 1), el $32,3 \%$ autoestima media $(0,35$ a 0,65$)$ y el $3,5 \%$ baja autoestima $(0$ a 0,34).

- El 57,7 \% de los alumnos del primer grado de educación secundaria de las instituciones educativas estatales del distrito de Huancayo tiene una baja comprensión de información en el área de Ciencia, Tecnología y Ambiente (0 a 10), el $23,9 \%$ comprensión media (11 a 15) y el $18,4 \%$ comprensión alta (16 a 20).

- Existe correlación significativa, directa y baja entre autoestima y comprensión de información en el área de Ciencia, Tecnología y Ambiente en los alumnos del primer grado de educación secundaria de las instituciones educativas estatales del distrito de Huancayo $(r=0,119)$.

\section{LITERATURA CITADA}

Branden, N. 1993. El poder de la Autoestima. Ediciones Paidos, Barcelona - España.

Chahuayo, A. y Díaz, B. 1993. Inventario de autoestima original forma escolar S. Coopersmith, Arequipa - Perú.

Córdova, M. 2003. Estadística inferencial. Edic. PUCP, Lima - Perú. 
De Zubiría, S.; et al. 1995. Tratado de pedagogía conceptual:operacionesintelectualesycreatividad, Fundación Alberto Merani para el Desarrollo de la Inteligencia, Fondo de Publicaciones Bernardo Herrera Merino, Colombia.

Domínguez, J. y Valentín, A. 2007. La técnica vivencial en la comprensión de información en las alumnas del segundo grado de secundaria del Área de Ciencia, Tecnología y Ambiente de la Institución Educativa Técnica María Inmaculada, Huancayo - Perú.

Homphreys, T. 1999. Autoestima para niños y padres; la clave para la educación, la salud emocional y el éxito escolar y humano de tus hijos, Neo Person Ediciones, Madrid - España.
Massone, A. y Gonzalez, G. s/f. Lectura: comprensión vs. retención de información. Una interpretación cognitiva. Revista Iberoamericana de Educación. Universidad Nacional de Mar del Plata, Argentina.

Ministerio de Educación. 2006. Orientaciones para el trabajo pedagógico; Área de Ciencia, Tecnología y Ambiente. Impreso por FIMART S.A.C. Lima Perú.

Ribeiro, L. 1997. Aumente su autoestima. Ediciones Urano, España.

Uculmuna, Ch. 2002. Autoestima. Lima - Perú.

Yataco De la Cruz, L. 2001. La autoestima escolar; la función tutorial del docente. Gráfica "Nelly" Distribuidora "J.C". Lima - Perú. 